\title{
Lassa fever is unheralded problem in West Africa
}

According to a new report from the Londonbased charity Merlin, poor laboratory services and a lack of understanding of the biology of the Lassa virus is preventing its containment in war-torn Sierra Leone, the hyperendemic center of the disease. "Without funds for basic diagnostic equipment, we cannot detect the disease satisfactorily," says Merlin's Tim Healing. Lassa fever has been estimated to cause 5,000 deaths annually in West Africa.

The charity, which advises the World Health Organization on the condition, based its report on an

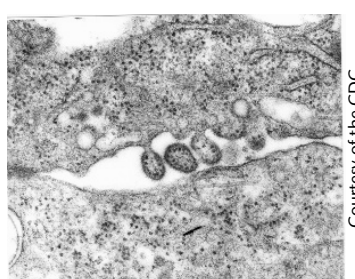
Lassa virus budding from cell membrane in tissue culture
University of Texas, Brownsville, which has shown success in non-human primates $(J$. Virol. 74, 6777; 2000)—has been stopped in its tracks by HIV. "Even though the vaccine is carried in a very attenuated strain of vaccinia, more attenuated than the vaccine that eradicated small pox, there are grave doubts that it would be usable in West Africa where there is so much HIV because people with low CD4 counts can develop disseminated vaccinia infection," explains McCormick.

McCormick's team is now researching other, more suitable vectors such as DNAbased vaccines either for assessment of Sierra Leone's
main treatment hospital in Kenema Around $16 \%$ of people admitted to hospitals in Sierra Leone and Liberia are estimated to have Lassa fever. Although PCR analysis can confirm the presence of virus within hours, hospitals in these areas seldom have access to such technology and, because the clinical symptoms of the disease are non-specific, physicians rely on differential diagnosis. They must first exclude other conditions such as tuberculosis and malaria before presuming infection with Lassa virus.

The disease is extremely rare in Western countries, thus only limited scientific research has gone into developing a treatment for the condition. Healing says that Lassa's low profile is exacerbated because of the Western media's focus on the more sensational hemorrhagic fever, Ebola. But while 150 people died of Ebola last year, 300,000 people died from Lassa.

Nevertheless, interest in Lassa virus in the United Kingdom has grown in recent months due to the death of a staff member from the Department for International Development and of several Red Cross workers from the disease within the last 12 months. "Sometimes Westerners need to be afflicted for serious diseases to be taken notice of," says Merlin Health Advisor, Jane Moore. Similar recent deaths in Germany have also sparked a pocket of research interest there.

Currently the anti-viral drug ribavirin is used to kill off the virus. Left untreated, patients can die within weeks from volume-deficiency shock due to uncontrollable heavy hemorrhage from all organs.

Vaccination is the only viable control measure for Lassa, yet further development of the most advanced vaccine- $-\mathrm{a}$ recombinant vaccinia vaccine developed by Joe McCormick and Sue Fisher-Hoch at the direct use or to be included in a yellow fever vector, which is less virulent. An added com-
According to the latest data from the US Centers for Disease Control and Prevention (CDC), even though heart disease and cancer still accounted for over half of all deaths in 1999, there was a $2 \%$ reduction in the numbers of mortality they caused compared with the previous year. This continues a downward trend for both diseases. However, more Americans are dying from diabetes (up by 3.3 $\%$ ) and respiratory diseases (up by $4 \%$ ), with the latter rise being sparked by two influenza outbreaks.

Increases were also seen in deaths due to septicemia (6.6\%), hypertension (5\%), while deaths due to HIV declined by $3.6 \%$ - a much smaller drop than was posted either of the last three years when the rate fell by $28.8,47.7$ and $20.6 \%$ respectively. Life expectancy estimates at birth in 1999 remained the same as in 1998, 76.7 years.

Meanwhile, a 34-year long study has raised questions about just how much exercise improves health. A panel of leading exercise scientists concluded that an inverse and mostly linear relationship exists between physical activity and the rate of allcause mortality, cardiovascular and coronary heart disease, type 2 diabetes and colon cancer. However, for many diseases the dose-response relationship is unclear based on a lack of, or contradictory, data. plication is that research funding for Lassa is hard to come by. "The problem is that we don't have this disease in the United States or the UK," he says, "so there's not a great deal of interest and companies won't touch it because even if there are over 100 million people infected in West Africa, they don't have any money to buy a vaccine. If companies are not going to make a meningococcal vaccine they're not going to make a Lassa vaccine."

McCormick was part of an international consortium that applied unsuccessfully to the European Union's Fifth Framework program for vaccine research funding last year. "In my experience the EU system is strange because you don't get a score or an explanation or anything if a grant is rejected," he says. He is now waiting to hear whether the US National Institutes of Health will fund the same proposal.

Karen Birmingham \& Georgina Kenyon, London

\section{Update on American death, disease and exercise}

I-Min Lee of Harvard Medical School, author of one of the articles published in the Medicine and Science in Sports and Exercise journal, says that for heart disease and allcause mortality, benefit was shown at lower levels of activity but this decreases at higher levels.

The effect of exercise on heart attack, stroke and cardiovascular-related death is a major component of a new study in patients with type 2 diabetes. The National Institutes of Health's Look AHEAD (Action for Health in Diabetes) study will randomize 5000 overweight volunteers with diabetes to a program promoting weight loss via a low fat, low calorie diet and they will gradually increase their activity levels to 30-35 minutes of brisk walking for 5-6 days per week. Volunteers will be followed for up to 11.5 years, making this the first long-term study looking at the effect of weight loss and physical activity in diabetic patients.

Stephen Horwitz, New York \& 